181

Triple Trouble: A Rare Case Report of PCDH19, Autism and Epilepsy in a 7-Year-Old Male Child

Sailaja Bysani, $M D^{\prime}$; Jusleen Kendhari, $M D^{2,3}$; and Cale Robert, $M S^{3,4}$

${ }^{1}$ Resident Physician, Department of Psychiatry, University of Missouri, Columbia, Missouri

2 Training Director, Child and Adolescent Psychiatry

Fellowship

${ }^{3}$ Assistant Professor of Clinical Psychiatry, University of Missouri, Columbia, Missouri

${ }^{4}$ Medical Student, Department of Psychiatry, University of Missouri, Columbia, Missouri

ABSTRACT: Background: A 7-year-old Caucasian male presented to the emergency department with worsening aggression andbehavioral problems. He was diagnosed with seizure disorder at 9 months old and was started on Keppra. He also had delayed developmental milestones including failure to roll until 6 months, and failure to crawl until after 9 months. At 16 months he was aggressive towards other children including unprovoked attacks of biting and assault. When he was 6yrs old he underwent genetic testing which showed positive for PCDH19 mutation. The most consistent feature of this condition is the early onset ofseizures between 3 months to 3 years of age. He has been on nine anti-seizure medications since his diagnosis. For behavioral problems, he was placed on Focalin, Ritalin, Adderall patch which did not seem to work. He was then on non-stimulants, Zoloft, Prozac, Effexor, Zyprexa and Abilify all with little success.

During this admission, patient's mother described increasing acts of severe physical violence toward multiple people leading to significant bruising. He has a known past psychiatric history of ASD, ADHD,ODD and PCDH19 mutation associated epilepsy. On admission, his medications included Buspirone $5 \mathrm{mg}$ BID, Olanzapine $7.5 \mathrm{mg}$ BID, Methylphenidate $7.5 \mathrm{mg}$, Ritalin $7.5 \mathrm{mg}$, Zonisamide $150 \mathrm{mg}$ qhs, Clonidine $0.1 \mathrm{mg}$ qhs. He had significant sensory processing difficulties and lack of communication with peers. He was continued on Clonidine $0.1 \mathrm{mg}$ po qhs, Methylphenidate $7.5 \mathrm{mg}$ po qam and at noon, Zonisamide $150 \mathrm{mg}$ daily. Tapered down Olanzapine and initiated him on Risperidone. His condition improved. He was referred to Thompson center for ABA therapy. He is more interactive and has a smile.

DISCUSSION: PCDH19 gene mutations have long since been known to cause epilepsy and behavioral disturbances in females. Males, on the other hand, present as asymptomatic transmitters. PCDH19 is a gene located on Chromosome $\mathrm{X}$ and is responsible for the formation of a protein known as protocadherin 19. This protein is especially important as it functions as a $\mathrm{Ca} 2+$ dependent cell adhesion in the brain. The PCDH19 mutation, however, impedes protein formation leading to epilepsy and behavioral disturbances. Approximately $90 \%$ of symptomatic females possess the mutated gene on one of their X chromosomes. Males similarly carry the mutation on their X chromosome, however are typically asymptomatic. A rare mosaic variant of PCHD19 mutations has been seen in symptomatic males.

CONCLUSION: Although it is more difficult to think in terms of ASD if a PCDH19 patient has coexisting psychiatric comorbidities like ODD andADHD, clinicians must be keenly aware of other mood disorders. Seizures do not respond well to medications. Family education, psychopharmacological treatment, ABA and CBT were successful in treating the patient. Little is known about the long-term sequela and prognosis of PCDH19 mutation in the male patient population and thus further research is warranted.

FUNDING ACKNOWLEDGEMENTS: No funding.

182

\section{Unintentional Overdose on Long-Acting Injectable Risperidone}

\author{
Saira Pasha, $M D^{\prime}$; and Shirin Schilling, $M D^{2}$ \\ ${ }^{1}$ Psychiatry Resident, Department of Psychiatry, \\ UCONN Health, Farmington, CT \\ ${ }^{2}$ Attending Psychiatrist, Department of Psychiatry, \\ UCONN Health, Farmington, CT
}

ABSTRACT: Study Objectives: Understand the mechanism and pharmacokinetics involved in an unintentional overdose of injectableantipsychotic medication; identify how to transition between long acting injectable antipsychotics; formulate steps to ensure patient safety in an event of an unintentional overdose of injectable antipsychotic

METHOD: Review of a case study involving a 47-year-old male, with history of schizoaffective disorder and previous episodes of loss of consciousness of unknown etiology, who was admitted to the inpatient psychiatry unit at UConn Health John Dempsey Hospital for stabilization of psychotic symptoms and monitoring of unintentional overdose after initially being admitted to the ICU. He was found unconscious at home following a period of days where he self-injected four of $37.5 \mathrm{mg}$ Risperidone injections (estimated $150 \mathrm{mg}$ ) along with limited adherence to prescribed oral clozapine, doxepin and lorazepam. He reported selfinjecting additional medication to treat paranoia, auditory, visual and olfactory hallucinations. In the ICU, he was evaluated by Toxicology and Neurology for loss of consciousness thought to be from seizures, with no clear outcome. He was awake and alert within 24 hours of medical admission but became agitated, hostile, and psychotic prompting psychiatric admission. When his 